\title{
A TEM study on pre-excystment cellular structures of Euplotes encysticus ${ }^{1,2}$
}

\author{
GU FUKANG AND JUNMEI XU ${ }^{3}$ \\ Department of Biology, East China Normal University, \\ Shanghai 200062, China
}

\begin{abstract}
Right before the excystment of an Euplotes encysticus sawtooth-like folds appeared among the pellicle plasmalemma, the inner and outer alveolar membranes were still sticking together, and were not distinguishable. Microtubular layers already formed at the sites beneath the dorsal cortical pellicle corresponding to vegetative cells, but they still proceed to be organized on the ventral structures. Cristae, highly-tangled with tubular-type structures, appeared on the mitochondria, and were morphologically similar to that of vegetative cells. In the cortical ciliatures, such as ciliary shafts, kinetosomes, surrounding fibrillar cirral baskets, and attached structures of ciliatures, etc., they are different from those in resting cysts which are degenerated or lost. All the ciliature microtubules of ciliary shafts are of the $9+2$ pattern, but the microtubule-like structure aggregates at triplet microtubule centers of many kinetosmes, are still under various stages of differentiation. Microtubules beneath the kinetosomal rows are of a developmentally elongated stage; crowded chromatins of various shapes and sizes are found in macronucleus, but there are no nuclear pores (formed by nuclear membrane as in resting cysts) on the nuclear
\end{abstract}

1. Dedicated to professor Zhen YAO's 80th Birthday.

2. This work was supported by a grant from the National Natural Science Foundation.

3. Corresponding author.

Abbreviations: AZM adoral zone of membranelles; BRU bristle unit; $\mathrm{CH}$ chromatin ; $\mathrm{CW}$ cyst wall; ECW ectocyst wall; ENW endocyst wall ; FVC frontal and ventral cirri; GRO groove; KB kinetosomal base bracket; KC inter-kinetosomal connection; KRMT microtubule ribbon beneath kinetosomal rows; MAC macronucleus; MIC micronucleus; MIT mitochondrion; MT microtubule; MTS microtubule-like structure aggregate; PCMT postciliary microtubule ribbon; PEL pellicle; TC transverse cirri. 
Pre-excystment cellular structures of Euplotse

membrane where these chromatins attached.

Key words: Euplotes encysticus, excystment, pellicle, kinetosome, macronucleus.

\section{INTRODUCTION}

In the studies of cysts and their differentiation of cellular structures in ciliates, many reports have been published on both light microscopic and SEM observations of kinetosome-resorbing cysts; especially deep-going observations with TEM were reported on the de-differentiation and re-differentiation of kinetosome- resorbing cyst of Gastrostyla steinii[1] and Histriculus muscorum[2], which have provided useful information on the differentiation and characteristics of their pattern formations in sub-microscopical structures of these ciliates under special conditions. Previously, only a few ciliates have been discovered for their formation of nonkinetosome-resorbing cysts; and for the study of their cysts, it was mainly on the differentiation of ciliatures and some structures during encystment and excystment stages on light microscopical and SEM levels[3-8]. For TEM works, there are reports on the formation of resting cyst structures on Diophrys scutum[9] and Euplotes muscicola[10]. Here, based on our former TEM observations of sub-microscopical sturctures on cortical cytoplasm, ciliatures and nuclear apparatus of resting cysts on Euplotes encysticus[11, 12], we have observed the differentiation characteristics of their pre-excystment cellular structures, hoped to provide new information for further study on their structural differentiation under special conditio5ns.

\section{MATERIAL AND METHOD}

\section{Material}

Clonal cultures of Euplotes encysticus (from the lake at the Shanghai Zoo, Spring, 1988), after segregation and feeding with Chilomonas paramecium were used in the experiments.

\section{Method}

The method of inducing $E$. encysticus into their encystments and excystments is: cultivate the cells around $25^{\circ} \mathrm{C}$, until they reach a certain density (about $15000 \mathrm{cells} / \mathrm{ml}$ ), 5 days later, cysts can be formed. Treating resting cysts with freshly filtrated pond-water, and excystment can be observed within 1.5-2.0 hours. Then, preparing TEM samples of cells before excystment (approx. $0.5 \mathrm{hr}$.), making TEM observations, taking photos, etc. For all the methods of cell development and inductions, TEM sample preparations, etc., refer to our previous reports[3, 4, 11].

\section{RESULTS}

\section{Cyst wall (CW) and non-ciliated cortex}

There are no apparent differences on the wall sturctures between pre-excystment cysts and those of resting cysts: their inner and outer walls remain intact, similar dense structures for the outer wall (ectocyst wall, ECW), and relatively lower 
electron- density for the inner wall (endocyst wall, ENW). Still, there are tiny particles in the space between them. Also, the protrusions formed by outward extension of ECW are still visible, and sawtooth-like folds appear on the pellicle (PEL) next to ENW, and with larger gaps (between PEL and CW) formed at certain intervals. Apparent single-membraned structures can be seen at the outer plasmalemma of the PEL, but it is difficult to distinguish the alveolar membranes of the inner and outer membranes (which stick together) at its inner rims. Beneath the PEL, it is already visible that every 3 microtubules (MTs) are composed of a triangular $(\therefore)$ unit which are arranged into a MT layer at certain intervals, corresponding to dorsal sub-PEL MT layers (Fig 1) at dorsal-ventral intersecting areas of future vegetative ciliates. In some TEM samples, MTs are grouping together under the PEL, forming MT layers at certain intervals (Fig 2), corresponding to the dorsal sub-PEL MT layer on the right side of dorsal cristae in vegetative cells.

At this stage, cortical mitochondria (MIT) seem greatly contracted, smaller than those of resting cells, and the cristae are tangled with tubular-type sturctures which appeared within the MIT. At this point, an apparent contrast formed between the MIT and their surrounding cytoplasm (Fig 2). MIT of similar morphology can be seen at the ciliated cortical areas and around the nuclear apparatus.

Fig 1. Cyst wall (ECW ectocyst wall, ENW endocyst wall), pellicle (big arrow), and subpellicle microtubule layer (small arrow) of pre-excystment $E$. encysticus.

Fig 2. Dorsal sub-pellicle microtubule layer (DPM) (arrow), mitochondrion (MIT) and dorsal bristle unit (BUR).

Fig 3. Cross section of AZM cilia and microtubule ribbons (arrow) at the rim of ciliature area.

Fig 4. Cross section of AZM membranelle kinetosomes, showing the tubular axis (arrow).

Fig 5. Cross section of AZM membranelle kinetosomes, showing the center of triplet microtubules without tubular structures (arrow), and the inter-kinetosomal connections (KC).

Fig 6. Sagittal section of AZM kinetosomes, showing the microtubule-like structure aggregates (MTS), inter-kinetosomal connections (KC) and attached structures at kinetosome bases (arrow).

Fig 7. Scatteringly distributed frontal and ventral cirri (FVC) and near by mitochondria (MIT).

Fig 8. Cross section of ciliary shafts within a cirrus.

Fig 9. 5 transverse cirri (TC) in the same cirral lumen.

Fig 10. Sagittal section of ciliary kinetosomes, showing the microtubule- like structures aggregate (MTS) and parasomal sac (arrow).

Fig 11. Microtubule ribbons beneath the kinetosomal rows (KRMT), postciliary microtubule ribbons (PCMT), and microtubules near the cirrus lumen (arrow).

Fig 12. Sagittal section of ciliary kinetosomes, showing the inter-kinetosome connections (KC) and kinetosomal base bracket (KB).

Fig 13. Showing the grooves (GRO).

Fig 14. Cyst wall (CW), grooves (GRO) and pellicle (PEL) (PM, plasmalemma; AV, alveolar membranes).

Fig 15. Sagittal section of bristle unit (BRU), showing developing ciliary shaft (arrow).

Fig 16. Macronucleus (MAC), chromatin (CH), and nucleolus (NU).

Fig 17. Micronucleus (MIC). 
Pre-excystment cellular structures of Euplotse
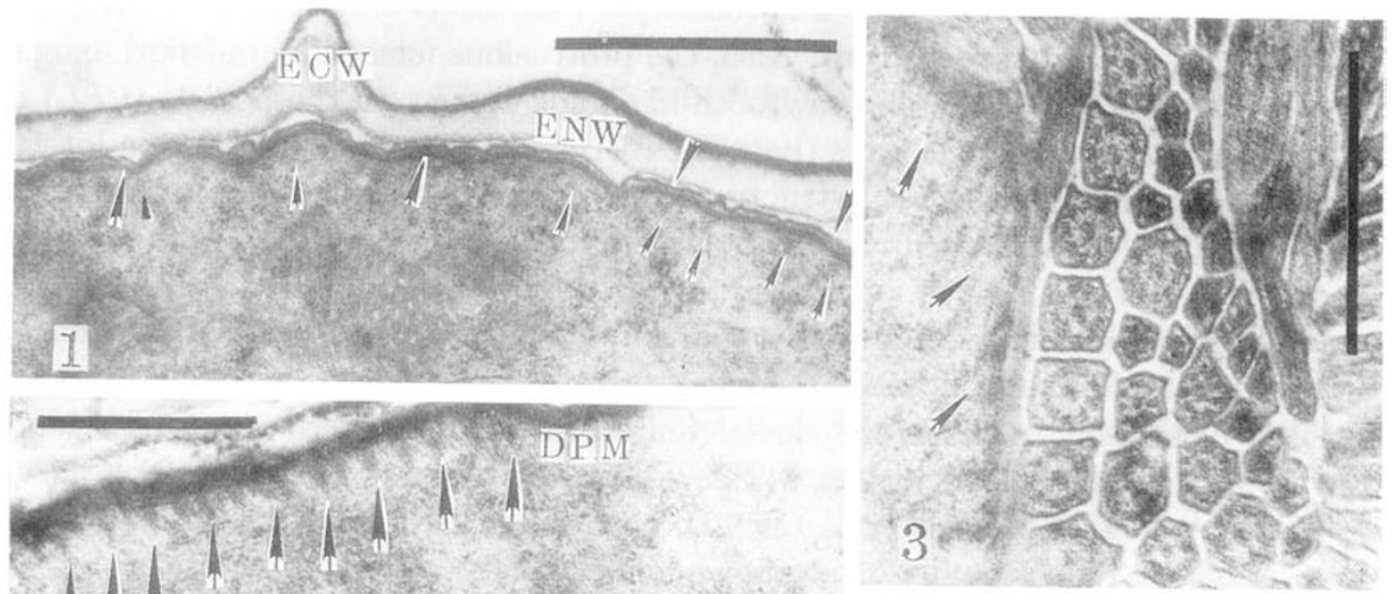

$k^{2}=1 \mid$

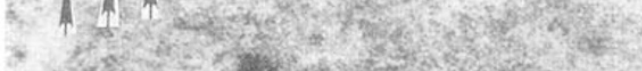

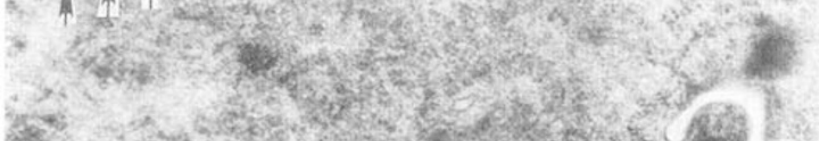

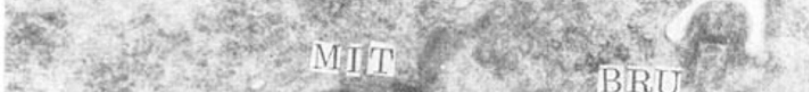

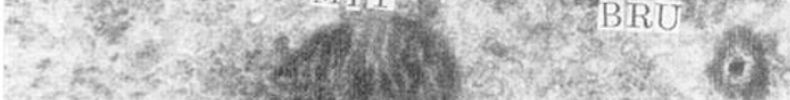

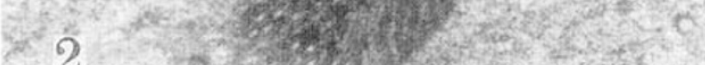

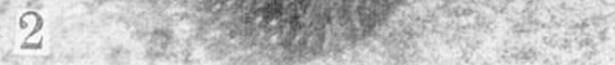

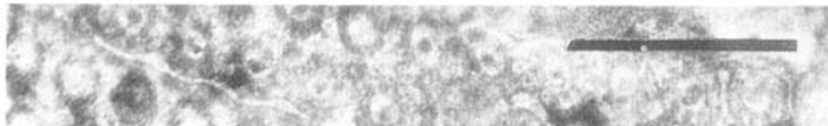

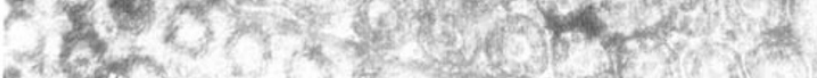

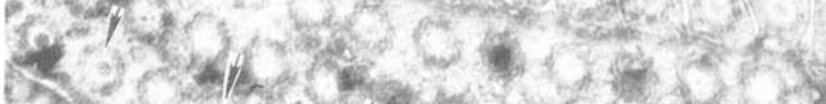
1.200.

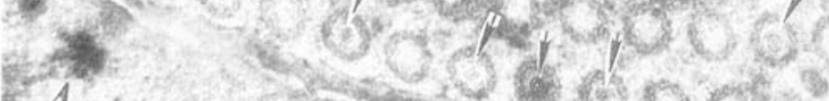
Q7. 4 if

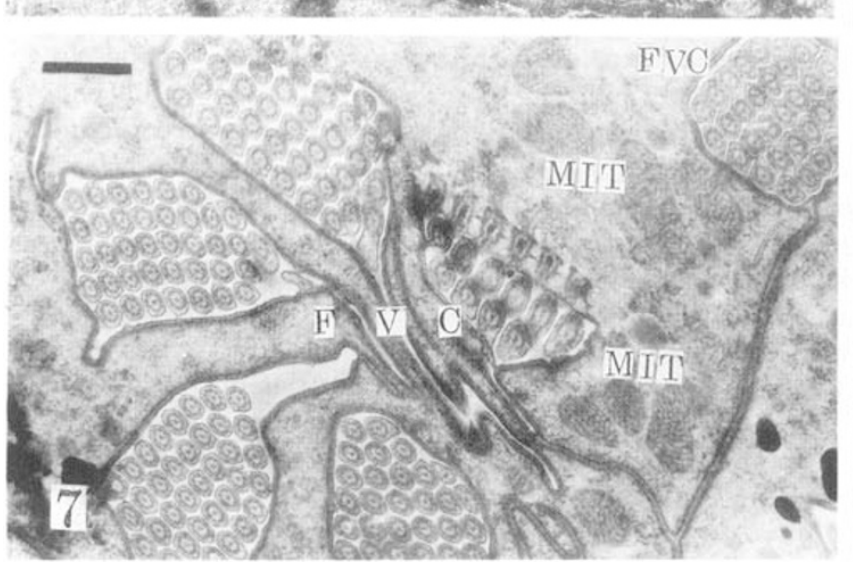

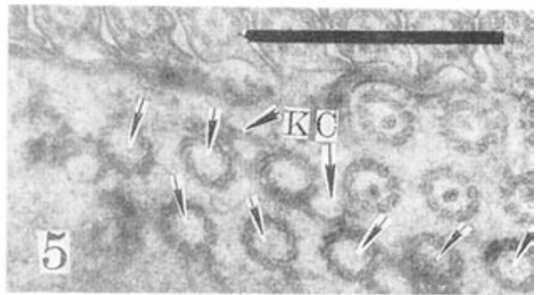

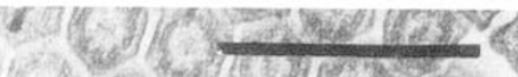

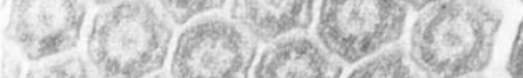

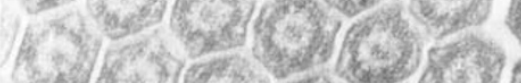
Q15)

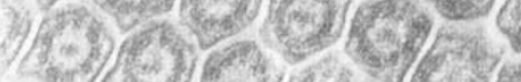

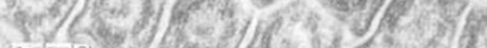
(7MTS

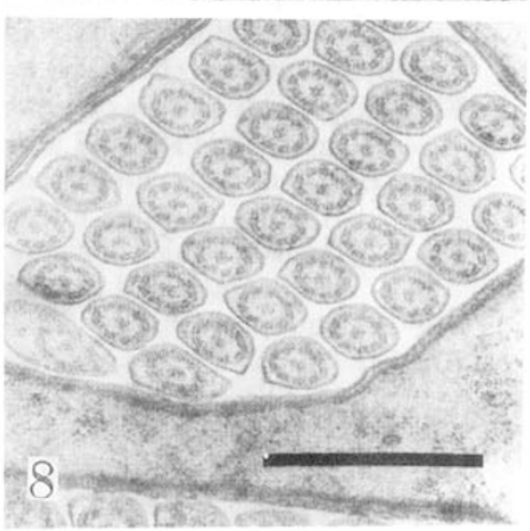


Gu FK and JM Xu
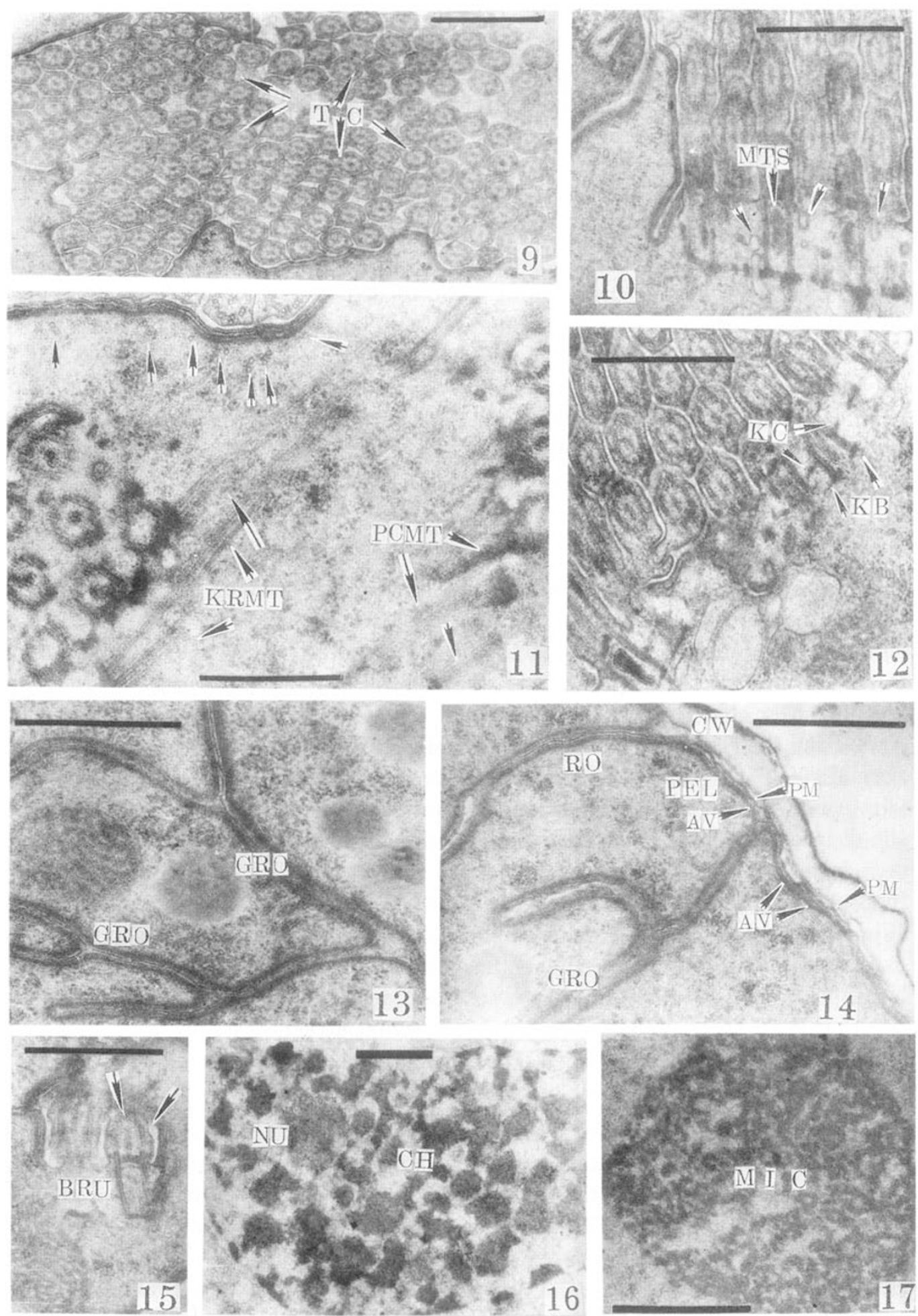
Pre-excystment cellular structures of Euplotse

\section{Ciliated cortex}

Adoral zone of membranelles (AZM): TEM samples on the anterior parts of AZM reveals that MT ribbons are densely distributed in peripheral areas. No degeneration and disintegration can be seen in the ciliatures. Anterior parts of the ciliary shafts hold closely together and are irregular polygonally-shaped. Their shape may be due to the rapid growth and development of many cilia within a narrow area (Fig 3). At the ciliary shafts' bases, both peripheral doublet MTs and central pair of MTs are of " $9+2$ " structure. Cross and sagittal sections of ciliary kinetosomes reveal that AZM membranelle units ( composed of 3 rows of kinetosomes) can be seen in the rectangular cavity surrounded by membraneous structures. Morphological differences can be seen at the center of some kinetosomal triplet MTs. There are less contrasted tubular axis structures within some of them, but they are not connected with slightly bent spokes among the triplets (Fig 4). Some of them have no tubular structures (Fig 5). Highly electron-dense, column-shaped structures, i.e., microtubule-like structure aggregates (MTS) have been found in some others (Fig 6). For attached kinetosomal structures, there are inter-kinetosomal connections (KC) and attached structures at the kinetosomal bases, etc. (Fig 5,6).

Ventral cirral area: Frontal and ventral cirri (FVC), which are closely grouped together in resting cells, are scatteringly distributed in this stage within the lumens surrounded by membraneous structures. Cirri lumens are connected with grooves (GRO) which are the extensions of their lateral membraneous structures. There are MIT near these lumens (Fig 7). Cilia of FVC have already been arranged into rows, both their peripheral doublet MTs and central pair of MTs are already in the normal "9+2" pattern (Fig 8). All transverse cirri (TC) are scatteringly distributed within one cavity (Fig 9). Cross and sagittal sections of ciliary kinetosomes reveal the disintegration and/or losing of partial kinetosomes in cirral cilia (which can be seen in resting cysts) no longer exists; but there are still aggregations with rather high electron density of MTS at the center of triplet MTs in most kinetosomes (Fig 10). Between every two kinetosomes and beneath the brackets (electron dense) extends a MT ribbon (MT ribbon benaeath the kinetosomal rows, KRMT) which horizontally goes into the anterior cytoplasm, and the neighboring MT ribbons are aggregated together (Fig 11). Other kinetosomal attached structures are: postcilliary MT ribbons (PCMT) (Fig 11), KC, kinetosomal base brackets (KB) (Fig 12), and parasomal sac (at the side of kinetosomes) (Fig 10).

As cirral cavites are formed by the sinking inward of cirri into the cell cytoplasm during cyst formation, the membraneous structures surrounding the cavities come from- the cell cortex of vegetative cells. At one side of the cavity, there are GRO (composed of two parallel PEL) (Fig 13) which extend out into the cell cytoplasm, up to CW, and merge into the cortex which sticks to CW (Fig 14). For PEL of some cirral lumens, plasma membrane and PEL alveolus can be distinguished already. Irregularly arranged MTs (one or more) can be seen at the peripheral areas near 
the cirrus lumen. This may be the future MT layer beneath the ventral PEL under re-organization and re- arrangement (Fig 11).

Dorsal bristle area: Dorsal bristle units (BRU) sank (with MT ribbons and MIT near by) into cavities surrounded by the PEL structures. At some BRU, there are rosette ciliary shafts under development and elongation (Fig 15). Highly electrondense central zones of triplet MTs within the BRU kinetosomes (Fig 2) may also be seen.

\section{Nucleus}

There are densely distributed chromatin $(\mathrm{CH})$ of various shapes and sizes within the macronucleus (MAC) (Fig 16). Some of the $\mathrm{CHs}$ attached to the inner nuclear membrane, but, different from that of resting cysts, no nuclear pores can be seen here. Very few nucleolus ( of larger size and lower electron density) can be seen within MAC. Micronucleus (MIC) (Fig 17) are situated near MAC, with CHs of similar size and high electron density.

\section{DISCUSSION}

According to the above-mentioned results, the differentiation of sub-microscopical structures in pre-excystment $E$. encysticus is not so complete as that of vegetative cells[12], but, compared to that of resting cysts, there are many characteristics (partial de-differentiation[11]) in the pre-excystment cells, i.e., many disintegrated and/or degenerated sturctures such as: sub-PEL MTs, partial cilia of AZM and FVC. In pre-excystment cells, MT ribbons of surrounding fibrilar cirral basket and cirral KRMT, etc., have already re-generated. As we have suggested [3], the phenomenon of sub-microscopical development which we have described in this paper is also a kind of cell structural re-differentiation based on their previous partially de-differentiated structures. The structural re-differentiation in a non-kinetosomeresorbing cyst is not the same as that of kinetosome-resorbing cyst. For the former, similar studies, as far as we are aware, have been scarce, further thorough explorations on their structural formation and control mechanisms are necessary.

During the pre-excystment stage, in E. encysticus, differentiation has already taken places on the PEL, MTs beneath PEL, cortical ciliatures and their attached structures, etc.; but, the completion of their differentiation and their final location should not be settled until the completion of its excystment. In the resting cells, MTS can be seen at the center of triplet MTs in the ciliary kinetosomes[11] while in pre-excystment cells, triplet MT centers still exist in some cilliary kinetosomes. However, in the same cell and /or the same ciliature, some of the kinetosomal triplet MT centers showing different type of tubular structure, or even tubular axis can be seen. Consequently, it is assumed that during their cyst formation, E. encysticus contracted rapidly, their ventral structures were folded inwardly and finally were surrounded by CW. Cell contraction is also accompanied by the contraction of vat- 
ions cellular structures and their closing to each other. Before the excystment of $\mathrm{E}$. encysticus, their contractile vacuoles are being activated first; then, other components of cyst-cytoplasm also begin to be activated. Hence, it might be assumed that the activity of the contractile vacuoles activates those still-resting cyst-cytoplasmic components, actuates them to react and flow, and results in the whole cell transfeting from the contracting status to an extending and activated status[3]. Thus, the MTS appearing at triplet MT centers of ciliary kinetosomes in resting cysts might be formed by the accompanied contraction of structures like tubular axis at the triplet MT center in corresponding kinetosomes. In other words, the so called "microtubule-like structure aggregates" are probably not genuine MTs, and the above-mentioned tubular axis are coming from structures like MTS. Perhaps, after excystment, all the tubular structures at the kinetosomal triplet centers in all ciliatures will morphologically be turned into normal forms.

In resting cysts of $E$. encysticus, among their cytoplasmic components, the mitochondrial tubular cristae swell already, with little apparently difference to the surrounding cytoplasmic structures. But, comparing this with the auto-digesting phenomenon of autophagic vacuoles, a certain process of material and energy may be still transfer is performed even within resting cysts[11]. In this paper, it is observed that there are many more MITs (beneath cell pellicle, around ciliatures, and near nuclear apparatus) in pre-excystment cells than there are in resting cyst. Furthermore, there clearly are highly-tangled tubular cristae on these MITs which are functional organelles for energy metabolism. Thus, it is assumed that the existence and morphological changes of the MIT in pre-excystment cells might be related to the extensive activities of material catabolism, energy metabolism, and structure anabolism which had taken place in these cells at this developmental stage.

Finally, it has been observed that in this stage, the MAC chromatin morphology is different from that of resting cysts, and, at the site where the $\mathrm{CH}$ are attached, no nuclear pore structures have been noted. Further exploration should be carried out on the mechanisms and significance of chromatin changes and the formation and disappearance of these nuclear pores during the period from encystment to excystment.

\section{REFERENCES}

[1] Walker GK, Maugel TK, Goode D. Encystment and excystment in hypotrich ciliate. I. Gastrostyla steinii. Protistologica 1980; 4:511-24.

[2] Matsusaka T, Nakamura T, Nagata K. Ultrastructure, disintegration and formation of a cirrus in the vegetative, encysting and excysting ciliate, Histriculus muscorum. J Electron Microsc 1984; 33:217-29.

[3] Gu FK, Zhang ZR. Differentiation of cilliature during encystment and excystment in Euplotes encysticus. Acta Zool Sin 1991; 37:287-92.

[4] Gu FK, Zhang ZR. Studies on the macronucleus and micronucleus during encystment and excystment in Euplotes encysticus. Acta Zool Sin 1992; 38:208-13.

[5] Matsusaka T, Noguchi 0, Yonezawa F. Cortical morphogenesis during encystment in a ciliate, Euplotes encysticus Yonezawa, 1985. Europ J Protistol 1989; 24:133-7. 


\section{Gu FK and JM Xu}

[6] Rawlinson NG, Gates MA. The encystment process in the ciliate Euplotes musdcola: an integrated light and scanning electron microscopic study. J Protozool 1985; 32:729-35.

[7] Yonezawa F. New hypotrichous ciliate Euplotes encysticus SP. nov. J Sci Hiroshima Univ Ser B Dev 1985; 32:34-45.

[8] Yonezawa F. Encystment progress and the relation of excystment to cell cycle in Euplotes encysticus (Ciliophora). J Sci Hiroshima Univ Ser B Dev 1 1985; 32:57-71.

[9] Walker GK, Maugel TK. Encystment and excystment in hypotrich ciliate. II. Diophrys scutum remarks on comparative features. Protistologica 1980; 16:525-31.

[10] Bussers JC, Hoesdorff M, Bolome M, Greco N, Gofflnet G. L'enkystement du cilie hypotriche Euplotes muscicola. Protistologica 1986; 22:457-60.

[11] Gu FK, Ni B. An ultrastructural study on resting cyst of Euplotes encysticus. Acta Biol Exper Sin 1995 (in press).

[12] Gu FK, Ji LM. An ultrastructural study on cortex and macronucleus of Euplotes encysticus. Zool Res 1995 (in press).

Received 14-3-1995. Revised 15-6-1995. Accepted 20-6-1995 